\title{
Protective Effect of Diploschistes ocellatus Against Heat Shock-Mediated Defects on Function of Reproductive Organs in Drosophila melanogaster
}

\author{
Mohammad Haddadi ${ }^{*}$, Javad Payam \\ ${ }^{1}$ Department of Biology, University of Zabol, Zabol, Iran
}

\author{
*Correspondence to \\ Mohammad Haddadi, \\ Emails: hadadimohamad@gmail.com, \\ m.haddadi@uoz.ac.ir
}

Received January 13, 2019 Accepted April 8, 2019 Published online June 30, 2019

\begin{abstract}
Introduction: Repeated heat shock (HS) stresses reduce the reproduction rate of Drosophila flies. Heat shock proteins (HSPs) protect cells against irreversible damages inducing heatinduced. Oxidative stress declines protective function of HSPs. Diploschistes ocellatus lichen aqueous extract possesses a strong antioxidant potential in vitro. Antioxidants can preserve HSPs function. Therefore, the present study for the first time investigated the cytoprotective effects of $D$. ocellatus aqueous extract against HS-mediated deleterious effects on reproductive function in Drosophila melanogaster.

Methods: Three different types of culture media including control, $30 \%$ lichen extract, and $60 \%$ lichen extract were prepared. Adult D. melanogaster flies were placed on Delcour medium and allowed to lay eggs for 2 hours. Then the eggs were equally distributed between the culture media. After flies completed their life cycle, the adult enclosed flies were exposed to HS. To assess reproductive function, the newly emerged adult flies were transferred to the freshly prepared regular culture medium every three days for 3 times and finally adult offspring born to these flies were enumerated.

Results: HS negatively affected the reproduction rate in flies in control group. Quantification of adult enclosed flies born to the D. ocellatus extract treated flies showed that lichen extract could negate the deleterious effects of HS on reproduction function of D. melanogaster in a dose-dependent manner.

Conclusion: Diploschistes ocellatus aqueous extract attenuated the harmful effects of HS stress on reproductive function of $D$. melanogaster. The secondary metabolites present in $D$. ocellatus can be considered as a bona fide candidate in novel drug development to target reproductive diseases in which oxidative stress is involved. Moreover, it can be concluded that $D$. melanogaster is an ideal model organism to induce cellular stress in vitro and study therapeutic potential of lichen extracts.

Keywords: Drosophila melanogaster, Diploschistes ocellatus, Heat shock proteins, Oxidative stress, Reproduction.
\end{abstract}

Please cite this article as follows: Haddadi $M$, Payam J. Protective effect of Diploschistes ocellatus against heat shockmediated defects on function of reproductive organs in Drosophila melanogaster. Int J Basic Sci Med. 2019;4(2):5155. doi:10.15171/ ijbms.2019.11.

\section{Introduction}

The subject of ecological impacts on quantitative hereditary parameters is generally concerned with the responses to the novel or stressful environment. ${ }^{1}$ Introduction of living beings to heat shock (HS), high temperature for a brief time-span, instigates a change in protein synthesis inside the cells. ${ }^{2}$ The natural significance of the HS response is evident from examination of survival among creatures presented directly to an extreme HS and those acclimatized by exposure to a gentle heat condition to facilitate synthesis
\end{abstract}

of stress proteins prior to exposure to a severe heat stress. ${ }^{3}$

All living organisms respond to elevated temperature as an external stress. ${ }^{4}$ To face these kinds of stresses, there are a number of cellular pathways to maintain homeostasis. One well-known group of these types of proteins are heat shock proteins (HSPs) that protect cells against damages induced by heat. ${ }^{5}$ HSPs act as signaling molecules and affect various cellular processes such as protein accumulation and trafficking, peptide transportation, and antigen processing. 6,7

(C) 2019 The Author(s); Published by Zabol University of Medical Sciences. This is an open-access article distributed under the terms of the Creative Commons Attribution License (http://creativecommons.org/licenses/by/4.0), which permits unrestricted use, distribution, and reproduction in any medium, provided the original work is properly cited. 
Antioxidant compounds attenuate free radicalinduced damages by affecting electron transport chain function and inhibiting oxidation of lipids and other macro molecules. ${ }^{8}$ HSPs possess antioxidant and antiinflammatory potentials. These proteins assist the primary and secondary folding of proteins and thus protect the cells against damages and apoptosis. ${ }^{9}$ It has been reported that elevation of cellular HSP level can cause resistance against different stresses. ${ }^{10}$ HSPs along with antioxidant molecules scavenge reactive oxygen species (ROS) in oxidative stress. ${ }^{11,12}$ Moreover, cellular antioxidants lead to a lower need for HSP expression to re-assemble the denatured proteins. ${ }^{13}$

Heat has severe effects on physiology and fitness of insects. For Drosophila melanogaster, extreme temperature is extremely harmful and even fatal. Due to lack of physiological processes for temperature balancing in these insects, they adjust their body temperature via different behavioral responses. ${ }^{14}$

In Drosophila, reactions to intense heat stress have been estimated as survival rate, sedation time or recovery from sedation, which are characteristics in which locomotor capacity is involved. ${ }^{15,16}$ However, to assess the unpleasant impacts of naturally occurring thermal stress, it is also possible to concentrate on reproductive features including fecundity, fertility, and hatchability. These reproductive features are regularly influenced by temperature changes which may be even evolutionarily more substantial. ${ }^{17}$

Survival and reproduction are two major parameters of fitness. Some studies have reported the negative impacts of HS on reproduction capacity of D. melanogaster. HS may introduce the cells to oxidative stress and thus antioxidants can ameliorate the deleterious effects of HS on reproductive health of fruit flies.

Lichens are distinctive types of living organisms composed of two elements, a fungus (namely, mycobiont) and an algae or cyanobacterium (called as photobiont). These two eukaryotic components live in symbiotic relationship. ${ }^{18-20}$ The fungi produce the thallus structure (the main body of lichen) that hosts the algae or the cyanobacterium, and in addition to protection, provides optimal conditions for photosynthesis of the photobionts. This procedure produces sugars and other nutrients required for growth of the fungus. ${ }^{20}$ As with plants, lichens are also able to synthesize secondary metabolites, and thus are used in folk medicine in many parts of the world. Most of these bioactive metabolites possess antioxidant, anti-bacterial, anti-inflammatory, and anti-cancer properties.

D. ocellatus is a wide-spread species of lichen growing on calcic rocks and soils. Its numerous thalli structures are powdery light grey to white. Each thick thallus encloses a black apothecium which is a disc-like composition on the exterior of the thallus and is used in sexual reproduction of the lichen. ${ }^{21}$

Lichens have been used as valuable biological resources since ancient times. People in different countries use the lichens as remedies, foods, and natural dyes. ${ }^{22,23}$ Lichens are also considered as natural therapeutics in pharmaceutical industry due to their unique bioactive ingredients. $^{24}$ Lichens possess secondary metabolites with very strong antioxidant potential that are capable of scavenging free radicals. ${ }^{25}$ In the present study, the cytoprotective effects of Diploschistes ocellatus aqueous extract were evaluated against deleterious actions of thermal stress on reproductive function of $D$. melanogaster for the first time.

\section{Materials and Methods}

Fly Stock and Husbandry

Wild type of $D$. melanogaster, Oregon $\mathrm{K}$ strain was obtained from Drosophila Lab, Department of Biology, University of Zabol, Zabol. Flies were raised, amplified, and maintained on standard wheat cream agar medium supplemented with dry yeast granules at $25 \pm 1^{\circ} \mathrm{C}$ and $50 \%-60 \%$ relative humidity in a vivarium. Synchronized adults were used in all the experiments.

\section{Lichen Aqueous Extract Preparation}

The D. ocellatus lichen was collected from local environment. Taxonomy of the lichen was confirmed by a botanist. The vegetative parts were removed and washed. Samples were air dried and powdered. A suspension was prepared in $100 \mathrm{~mL}$ distilled water and kept in shaker incubator at $37^{\circ} \mathrm{C}$ overnight. Finally, the solution was filtered using Whatman filter paper grade 1 and used for the experiments.

Evaluation of Antioxidant Potential, Toxicity, and Effective Dose of Diploschistes ocellatus extract

The antioxidant activity $D$. ocellatus aqueous extract was assessed by DPPH radical scavenging assay as described by Brand-Williams et al. ${ }^{26}$ with minor modifications.

To determine possible lethality of $D$. ocellatus extract, different fly culture media were prepared with $10 \%, 50 \%$, and $90 \%$ D. ocellatus extract concentrations. A total of 20 five-day-old adult flies were transferred to each medium vial and were monitored for 3 weeks. Flies were shifted to a new medium vial with same preparations every 4 days. Number of dead flies was recorded each day.

\section{Diploschistes ocellatus Extract Treatment}

Following proliferation of flies, virgin females and single males were isolated and kept in separate culture vials. On the day 5, 50 males and 30 females were transferred to culture bottles containing 3 different media, 60\% (v/v) D. ocellatus extract, $30 \%(\mathrm{v} / \mathrm{v})$ D. ocellatus extract, and control media (without extract). The flies were allowed to mate and reproduce for 3 days and then the adult flies were discarded. The emerging offspring were taken for HS experiments. 


\section{Heat Shock Exposure}

The newly enclosed adults were separated based on their gender and transferred to freshly prepared culture media vials. Two-day-old virgin female flies and single males were transferred to agar media vial to avoid lethality due to desiccation and then kept in oven at $37^{\circ} \mathrm{C}$ for 90 minutes. After a16-hour rest in regular medium in vivarium, the flies were added to agar culture vials and again were exposed to $40^{\circ} \mathrm{C}$ for 60 minutes. ${ }^{3}$

Reproductive Success Assessment

Following a 3-hour rest interval after HS exposure, the 30 males and 20 female flies were transferred to normal culture bottles and were allowed to copulate and lay eggs for 3 days. Then adult flies were discarded. The culture bottles were carefully monitored for nine days to quantify the egg-to-adult number of flies. Indeed, the number of hatched larvae that could go for pupation was included by counting emerging adult flies. This procedure was done repeatedly every 3 days. ${ }^{27}$

\section{Statistical Analysis}

The data was presented as mean \pm standard error (SE) and analyzed by independent samples student t-test and one-way ANOVA followed by Dunnet test in PASW (predictive analytics software) version 19.0. The $P$ value of $<0.05$ was considered as significance level.

\section{Results}

Diploschistes ocellatus Aqueous Extract Antioxidant Activity, Toxicity, and Dose Selection

The antioxidant activity percentage (AA\%) of D. ocellatus aqueous extract was found to be 84 (inhibition \%) according to the DPPH scavenging activity of ascorbic acid. This value indicates the high antioxidant potential of the lichen extract.

The extract was not toxic and caused no lethality at any of the doses used. Therefore, two v/v concentrations (30\% and $60 \%$ ) of D. ocellatus aqueous were chosen for the main experiments as effective doses.

Effect of Heat Shock on Flies' Reproduction Success To test the protective potential of D. ocellatus aqueous extract against reproduction failure following HS in flies, the number of enclosed adult offspring of parents under different culture conditions was determined. As shown in Table 1, HS stress adversely affected the reproduction rate in control group (i.e. the flies that had not been treated with D. ocellatus aqueous extract). Though the number of offspring dramatically reduced over the 9-day screening, the most severe effect was found to be in the first 3 days after HS stress.

Effect of Diploschistes ocellatus Aqueous Extract on HSInduced Reproduction Defects

Quantification of enclosed adult flies born to D. ocellatus treated parent flies showed that D. ocellatus could suppress the deleterious effects of HS on reproduction success. As indicated in the Table 2, D. ocellatus extract exhibited a dose-dependent protective effect on reproductive success (i.e. the effects were more pronounced in $60 \%$ D. ocellatus extract compared to the $30 \%$ D. ocellatus extract).

In the absence of HS stress, lichen extract treatment alone did not change reproduction rate of flies. Comparison between control group and sham group who were treated with $D$. ocellatus extract but were not exposed to HS stress revealed no significant differences in the number of offspring born to D. ocellatus treated and control parent flies $(P=0.63$, Table 3$)$.

\section{Discussion}

The present study is the first report on the inhibition of the HS stress-induced cellular and molecular damages and restoration of their reproductive success to the normal level by the aqueous extract of $D$. ocellatus lichen. The fecundity, fertility, hatchability, and adult enclosure were found to be the main parameters affected by HS stress in this study. The number of enclosed adult flies that were able to reproduce was also determined. It was evident that D. ocellatus remarkably attenuated reproduction success of HS exposed flies.

Assessment of resistance to environmental stresses may not always be an appropriate strategy in terms of survival and reproduction. ${ }^{16,17}$ But in Drosophila species that are thought to have a short normal life span, ${ }^{26,28}$ even reversible damage may affect the reproductive success of the insect.

Temperature is an important factor in Drosophila life cycle and many physiological functions. ${ }^{29}$ There are reports on declined reproduction success in male fruit

Table 1. Comparison Between Offspring of Drosophila melanogaster Born to Sham and Control Groups

\begin{tabular}{lccc}
\hline \multirow{2}{*}{ Treatment type } & \multicolumn{3}{c}{ Number of Enclosed Adult Flies After Heat Shock $^{\mathbf{a}}$} \\
\cline { 2 - 4 } & $\mathbf{3}$ days & $\mathbf{6}$ days & $\mathbf{9}$ days \\
\hline Sham $(-H . S$, -Do) & $334 \pm 3$ & $312 \pm 2$ & $313 \pm 1$ \\
Control (+H.S, -Do) & $82 \pm 2$ & $132 \pm 5$ & $126 \pm 4$ \\
$P$ value & $<0.01$ & $<0.01$ & $<0.01$ \\
\hline
\end{tabular}

${ }^{a}$-Heat shock: The flies under $37^{\circ} \mathrm{C}$ for 90 minutes followed by a 16 -hour rest in regular condition; + Heat shock: The flies under $40{ }^{\circ} \mathrm{C}$ for 60 minutes.

Do, D. ocellatus. 
Table 2. Diploschistes ocellatus Aqueous Extract Protection Against Heat Shock Mediated Reproduction Defects in Drosophila melanogaster

\begin{tabular}{|c|c|c|c|}
\hline \multirow{2}{*}{ Treatment type } & \multicolumn{3}{|c|}{ Number of Enclosed Adult Flies at Different Intervals After Heat Shock ${ }^{a}$} \\
\hline & 3 days & 6 days & 9 days \\
\hline Control (-H.S, -D.o) & $109 \pm 4$ & $150 \pm 3$ & $139 \pm 3$ \\
\hline Treated (-H.S, + 30\% D.o) & $223 \pm 2$ & $342 \pm 3$ & $250 \pm 2$ \\
\hline Treated (-H.S, + 60\% D.o) & $315 \pm 3$ & $300 \pm 4$ & $320 \pm 2$ \\
\hline$P$ value & $<0.001$ & $<0.01$ & $<0.01$ \\
\hline
\end{tabular}

a -Heat shock: The flies under $37^{\circ} \mathrm{C}$ for 90 minutes followed by a 16 -hour rest in regular condition; +Heat shock: The flies under $40^{\circ} \mathrm{C}$ for 60 minutes.

Do, D. ocellatus.

Table 3. The Effect of Diploschistes ocellatus Aqueous Extract on Reproduction Rates of Control Flies (Not Exposed to Heat Shock)

\begin{tabular}{|c|c|c|c|}
\hline \multirow{2}{*}{ Treatment type } & \multicolumn{3}{|c|}{ Number of Enclosed Adult Flies } \\
\hline & 3 days & 6 days & 9 days \\
\hline Control (-H.Sa, - D.o) & $324 \pm 14$ & $304 \pm 2$ & $311 \pm 2$ \\
\hline Treated (-H.S, + 30\% D.o) & $309 \pm 2$ & $312 \pm 2$ & $307 \pm 4$ \\
\hline Treated (-H.S, +60\% D.o) & $317 \pm 2$ & $308 \pm 1$ & $302 \pm 3$ \\
\hline$P$ value & 0.68 & 0.58 & 0.7 \\
\hline
\end{tabular}

a -Heat shock: The flies under $37^{\circ} \mathrm{C}$ for 90 minutes followed by a 16 -hour rest in regular condition; + Heat shock: The flies under $40^{\circ} \mathrm{C}$ for 60 minutes.

Do, D. ocellatus.

flies exposed to prolonged high $\left(29^{\circ} \mathrm{C}\right)$ temperature. ${ }^{30}$ However, female flies are more sensitive to HS, and thermal stress can affect their adaptive parameters like mating tendency, fecundity, and fertility. ${ }^{31}$

High temperature and oxidative stress primarily induce HSPs expression. ${ }^{32}$ Moreover, severe oxidative stress impairs HSPs function. ${ }^{33}$ Association of these factors in vivo has been definitely confirmed. In addition to lacking complex physiological mechanisms to maintain body temperature in these tiny insects, simple simulation circumstances for environmental stress factors affecting Drosophila life in laboratory conditions facilitate systematic study of cellular responses to stressful stimuli.

With regards to the association between HSPs and oxidative stress, ${ }^{31}$ it is advisable to study cytoprotective potential of natural antioxidants, which are able to ameliorate such damages to cells. In addition, the role of secondary metabolites of lichens has not yet been extensively studied. These agents can have potential therapeutic properties due to their strong antioxidant capacities. ${ }^{32}$ Therefore, in the present study, the protective effects of $D$. ocellatus aqueous extract against functional damages induced by HS on reproduction rate of the fruit flies were investigated. The findings were in favor of protective role of $D$. ocellatus extract through restoration of reproduction of the flies exposed to HS.

Though antioxidant action of $D$. ocellatus could be the main reason for its cytoprotective impacts, molecular investigations on interaction between lichen extract components and HSPs are highly needed. Other therapeutic actions of the lichen, such as antiinflammatory, anti-cancer, and antibacterial, can be investigated in future investigations to extend limited knowledge about cellular mechanisms through which the D. ocellatus extract overcomes the external cell stressors.

\section{Conclusion}

Given the involvement of environmental factors and oxidative stress in development of wide spectrum of human diseases, and disease condition modeling in fruit flies, ${ }^{34}$ special attention can be directed to lichen secondary metabolites in future drug development studies. Nevertheless, aqueous extract of the D. ocellatus could be used as a solution containing numerous antioxidant compounds. Thus, characterization of molecules present in this solution is highly recommended. Moreover, recently characterized molecules will maintain the main status in drug development in future.

\section{Competing Interest}

The authors declare that they have no competing financial, professional, or personal interests that might have influenced the performance or presentation of the study described in this manuscript.

\section{Ethical Approval}

Not applicable.

\section{Acknowledgements}

The author gratefully thanks The Research and Technology Vice-chancellor of the University of Zabol. This work was supported by grant no. 9517-51. We also thank Mr. Faramarz Dahmardeh and Ms. Masoumeh Masoudinia for lichen collection and characterization. 


\section{References}

1. Hoffmann AA, Parsons PA. Evolutionary genetics and environmental stress. Oxford University Press; 1991.

2. Feder ME, Hofmann GE. Heat-shock proteins, molecular chaperones, and the stress response: evolutionary and ecological physiology. Annu Rev Physiol. 1999;61:243-282. doi:10.1146/annurev.physiol.61.1.243

3. Sisodia S, Singh BN. Effect of exposure to short-term heat stress on survival and fecundity in Drosophila ananassae. Can J Zool. 2006;84(6):895-899. doi:10.1139/z06-075

4. Peters EM, Smith M, Docrat A, Nadar A, Chetty K, Passmore JAS. The effects of a natural anti-inflammatory product on systemic markers of inflammation following downhill running: 2236. Med Sci Sports Exerc. 2009;41(5):278. doi:10.1249/01.MSS.0000355401.95790.36

5. Carey D, Pliego G, Raymond R. How endurance athletes breathe during incremental exercise to fatigue: Interaction of tidal volume and frequency. J Exerc Physiol Online. 2008;11(4):44-51.

6. Zhu J, Quyyumi AA, Wu $\mathrm{H}$, et al. Increased serum levels of heat shock protein 70 are associated with low risk of coronary artery disease. Arterioscler Thromb Vasc Biol. 2003;23(6):1055-1059. doi:10.1161/01. atv.0000074899.60898.fd

7. Chen B, Wagner A. Hsp90 is important for fecundity, longevity, and buffering of cryptic deleterious variation in wild fly populations. BMC Evol Biol. 2012;12:25. doi:10.1186/1471-2148-12-25

8. Poljšak B, Fink R. The protective role of antioxidants in the defence against ROS/RNS-mediated environmental pollution. Oxid Med Cell Longev. 2014;2014:671539. doi:10.1155/2014/671539

9. Hooper PL, Hooper JJ. Loss of defense against stress: diabetes and heat shock proteins. Diabetes Technol Ther. 2005;7(1):204-208. doi:10.1089/dia.2005.7.204

10. Rahimi M, Asgari AR, Khoshbaten A. the role of exercise preconditioning in cardioprotection against ischemia reperfusion injury. Physiology and Pharmacology. 2014;18(2):122-43. [Persian].

11. Ikwegbue PC, Masamba P, Oyinloye BE, Kappo AP. Roles of heat shock proteins in apoptosis, oxidative stress, human inflammatory diseases, and cancer. Pharmaceuticals (Basel). 2017;11(1). doi:10.3390/ph11010002

12. Wu CW, Biggar KK, Zhang J, et al. Induction of antioxidant and heat shock protein responses during torpor in the gray mouse lemur, Microcebus murinus. Genomics Proteomics Bioinformatics. 2015;13(2):119-126. doi:10.1016/j. gpb.2015.03.004

13. Booth FW, Thomason DB. Molecular and cellular adaptation of muscle in response to exercise: perspectives of various models. Physiol Rev. 1991;71(2):541-585. doi:10.1152/physrev.1991.71.2.541

14. Dillon ME, Wang G, Garrity PA, Huey RB. Review: Thermal preference in Drosophila. J Therm Biol. 2009;34(3):109119. doi:10.1016/j.jtherbio.2008.11.007

15. Gibert P, Moreteau B, Pétavy G, Karan D, David JR. Chill-coma tolerance, a major climatic adaptation among Drosophila species. Evolution. 2001;55(5):1063-1068. doi:10.1111/j.0014-3820.2001.tb00623.x

16. Hoffmann AA, Sørensen JG, Loeschcke V. Adaptation of Drosophila to temperature extremes: bringing together quantitative and molecular approaches. J Therm Biol. 2003;28(3):175-216. doi:10.1016/S0306-4565(02)00057-8

17. Fasolo AG, Krebs RA. A comparison of behavioural change in Drosophila during exposure to thermal stress. Biol J Linn Soc. 2004;83(2):197-205. doi:10.1111/j.10958312.2004.00380.x

18. Wasser SP, Nevo E. Lichen-forming, lichenicolous, and allied fungi of Israel. Ruggell. Gantner Verlag; 2005:1-384.

19. Ghazanfar SA, Fisher M. Vegetation of the Arabian peninsula. Springer Science \& Business Media; 2013.

20. Nash TH. Lichen biology. Cambridge University Press; 1996.

21. Ahmadjian V. The lichen symbiosis. John Wiley \& Sons; 1993.

22. Sharnoff SD. Lowly lichens offer beauty and food, drugs and perfume. Smithsonian. 1984; 15:135-41.

23. Varnam AH, Evans MG. Environmental microbiology. Manson Publishing; 2000.

24. Gayathri D, Swamy CT. Lichens: a novel and potential source as antimicrobials for human use. J Phytol. 2012;4(1):38-43.

25. Brand-Williams W, Cuvelier ME, Berset C. Use of a free radical method to evaluate antioxidant activity. LWT Food Sci Technol. 1995;28(1):25-30. doi:10.1016/S00236438(95)80008-5

26. Rosewell J, Shorrocks B. The implication of survival rates in natural populations of Drosophila: capturerecapture experiments on domestic species. Biol J Linn Soc. 2008;32(4):373-384. doi:10.1111/j.1095-8312.1987. tb00438.x

27. Nguyen TT, Moehring AJ. Cross-generational comparison of reproductive success in recently caught strains of Drosophila melanogaster. BMC Evol Biol. 2017;17(1):41. doi:10.1186/s12862-017-0887-1

28. Turelli M, Hoffmann AA. Cytoplasmic incompatibility in Drosophila simulans: dynamics and parameter estimates from natural populations. Genetics. 1995;140(4):13191338.

29. Rezaei A. The effect of different temperature on the viability and fertility of Drosophila melanogaster. Egypt Acad J Biol Sci. 2012;5(1):80-87. doi:10.21608/eajbsa.2012.14911

30. Schnebel EM, Grossfield J. Oviposition temperature range in four Drosophila species triads from different ecological backgrounds. Am Midl Nat. 1986;116(1):25-35. doi:10.2307/2425934

31. Ikwegbue PC, Masamba P, Oyinloye BE, Kappo AP. Roles of heat shock proteins in apoptosis, oxidative stress, human inflammatory diseases, and cancer. Pharmaceuticals (Basel). 2017;11(1). doi:10.3390/ph11010002

32. Adachi M, Liu Y, Fujii K, et al. Oxidative stress impairs the heat stress response and delays unfolded protein recovery. PLoS One. 2009;4(11):e7719. doi:10.1371/journal. pone. 0007719

33. Fernández-Moriano C, Gómez-Serranillos MP, Crespo A. Antioxidant potential of lichen species and their secondary metabolites. A systematic review. Pharm Biol. 2016;54(1):1-17. doi:10.3109/13880209.2014.1003354

34. Haddadi M. Drosophila melanogaster as a model to study human neurodegenerative diseases. Int J Basic Sci Med. 2018;3(1):9-12. doi:10.15171/ijbsm.2018.02 